\title{
Reseña de Experiencias didácticas para la innovación en el aprendizaje histórico escolar
}

Review of Experiencias didácticas para la innovación en el aprendizaje histórico escolar

Carlos D. Ciriza-Mendívil (*)

Editores: Gabriela Vásquez Leyton, Paula Soto Lillo, Cinthia Peña Hurtado, Óscar Valenzuela Flores

Título: Experiencias didácticas para la innovación en el aprendizaje histórico escolar

Edición: Ediciones Universitarias de Valparaíso

Lugar de Publicación: Valparaíso (Chile)

Año: 2020

Idioma: Español

ISBN: 978-956-17-0874-7

Páginas: 249

Este I ibro se pr esenta como un compendio de pr opuestas, un manual práctico de investigación-acción en el q ue di stintos profesores en formación desa rrollan propuestas didácticas innovadoras para el aula de historia en centros educativos de la región de Valparaíso en Chile. A nivel general, se trata de una obra cuyo objetivo principal es el desa rrollo de est rategias, prácticas y recursos de innovación doce nte así co mo su análisis y evaluación en I a práctica en aras de u n a prendizaje m ás significativo de la historia. En definitiva, como señala la propia obra, "enseñar menos y aprender más" historia debe ser el objetivo de todos nuestros procesos de enseñanzaaprendizaje de la disciplina, especialmente, a través de un aprendizaje competencial. Una educación histórica que pone, en esta obra, el foco principal en el desarrollo del pensamiento histórico que dote al alumnado de instrumentos de analisis, comprension o interpretación que le permitan abordar el estudio de la historia de forma autónoma y construir su propia representación del pasado, al mismo tiempo que adquirir un rol activo en el aula y en su propio aprendizaje.

Si bien el contexto en el que las propuestas didácticas de estas obra se desarrollan es específico -Valparaiso ( Chile)- el m arco cu rricular so bre el que se i mplementan es amplio e incluyen propuestas para alumnos de la asignatura de historia entre $7^{\circ}$ básico

*, Universidad Pública de Navarra (UPNA) carlos.ciriza@unavarra.es. ORCID: 0000-0002-2193-5978

Ciriza-Mendívil, C., (2021). Reseña de Experiencias didácticas para la innovación en el aprendizaje histórico escolar. Clio. History and istory Teaching, 47, 334-338. https://doi.org/10.26754/ojs clio/clio.2021476043 Recibido: 13/10/2021 Aceptado: 30/11/2021. 
y $4^{\circ}$ medio (12 a 17 años). Un enfoque curricular amplio que permite observar cómo el curriculum chileno no solo no limita el desarrollo del pensamiento histórico, sino que llega incluso a permitir, como muestra la obra, su desarrollo pormenorizado en distintos niveles y grados de dificultad.

De esta manera, a lo largo de esta obra se observa una mirada armonizada ent re teoría pedagógica y ejercicio profesional docente gracias al desarrollo de propuestas didácticas que salen de la práctica más habitual. Un total de siete propuestas divididas en capítulos en los que se desarrolla una nuva mirada hacia el curriculum y las temáticas, una reflexión más profunda de las fuentes históricas y su trasposición didáctica, pero también, un enfoque más amplio de los recursos y las tecnologías que se pueden aplicar a la docencia de la historia.

En el primer capítulo, titulado "Educación para la equidad: aprendizaje en la asignatura de historia en perspectiva de género", la propuesta de la autora Silva Andrade plantea una renovación en torno al curriculum. Así, su objetivo es reducir el foco androcéntrico del curriculum chileno y de sus planes de estudio a través de la incorporación del rol femenino en la historia en la asignatura de historia de primero medio. Los estudiantes deben en est a propuesta idefntificar preconceptos sobre género du rante el siglo $\mathrm{XX}$ para anal izar su desaparición o per manencia, confrontando realidades pasadas con situaciones del presente.

En el segundo capítulo, obra de Eugenin Rosales, Ramírez Ubilla y Ramos Olivares, titulado "Historia, cómic y educación: propuesta e implementación del trabajo con cómics en historia", comienza un apartado de la obra en el que las propuestas se centran en la valoración de los recursos didácticos que se utilizan en el aula. En esta propuesta, el objetivo principal es la valoración del cómic y su desarrollo en el aula de historia como una f uente primaria, en par ticular, ent re estudiantes de pr imero y segundo m edio en I a t emática del mundo ent reguerras y el totalitarismo. El t ercer capítulo de este libro, continúa el enfoque abierto por el anterior capítulo, esto es, el desarrollo y valoración en el aula de nuevos recursos didácticos. Titulado "Enseñando historia a t ravés del a fihce pol ítico: experiencia doce nte desd e l a ense ñanza de I a transición a la democracia en Chile" este capítulo de Neves Guzmán, desarrolla una propuesta apl icable a I os estudiantes desde $7^{\circ}$ bási co a $1^{\circ}$ m edio a través de la utilización del afiche político como herramienta y fuente primaria para el desarrollo del 
pensamiento hi stórico en el discente. $\mathrm{P}$ ara I a aut ora, est e t ipo de $r$ ecurso es especialmente i mportante al a hor a desa rrollar determinados elementos del pensamiento hi stórico tales como la imaginación histórica, I a r epresentación de la historia, I a i nterpretación histórica y I a co nsciencia hi stórico-temporal. Así, su propuesta evidencia cómo los estudiantes pueden alcanzar niveles de análisis profundos sobre el $\mathrm{s}$ ubtexto de est as fuentes históricas, esp ecialmente en I o ideológico, desarrollando un apr endizaje mucho más significativo de I a historia y del contexto en el que estos recursos se crearon.

Con el cuarto ca pítulo, co mienza un apar tado de I a obr a ce ntrado menos en I os recursos didácticos y $m$ ás en las estrategias desarrolladas en l a ense ñanza de I a historia y sus soportes. Este capítulo, obra de Ibáñez Orihuela, Zuñiga Zuñiga y Urzúa Zuñiga, se titula "Proyecto ABPP: propuesta didáctica con dispositivos móviles en la enseñanza de I a Historia, G eografía y Cie ncias Sociales" y propone I a fusión de I Aprendizaje Basado en Problemas con el uso de la aplicación para el telefóno móvil EDMODO. Esta propuesta se desarrolla para $3^{\circ} \circ 4^{\circ}$ medio en la asignatura del plan diferenciado Humanista y se centra en el análisis de la ciudad contemporánea como una "ciudad viva" a través de la aplicación y, especialmente, en l o que se refiere a la problemática de los incencios de Valparíaso en 2014. De esta manera, se incorporan las tecnologías al aul a, pero adem ás se fomenta el de sarrollo de I apr endizaje colaborativo y la ut ilización r esponsable de I as redes a través de una ci udadanía digital. El quinto capítulo continúa el desarrollo de distintas estrategias y soportes, en este caso, centrándose directamente en la recreación del trabajo del historiador. Este capítulo, $\mathrm{t}$ itulado "Para un est udio de I a historia antigua y los ciudadanos de hoy: utilización de fuentes históricas e implementación de est rategias didacticas en el aula para el desa rrollo del pensa miento hi stórico y la formación ci udadana" combina dos elementos. De una parte, el desarrollo del currículo histórico de $7^{\circ}$ básico y su temática en torno a la Historia Antigua y el pensamiento clásico. $Y$ de otra parte, el uso de fuentes históricas antiguas y presentes, desarrollando un juego de analogías entre el pasado y el presente en el que el canon de cultura clásico hace las veces de vínculo conector. De esta manera, la propuesta fomenta no solo el pensamiento histórico y sus distintos elementos, sino que también permite un aumento sustancial de la motivación del alumnado en torno a esta temática histórica a través de la analogía con el presente tanto artístico, como cultural y social en el concepto de la ciudadanía. 
El sexto capítulo continúa en la línea de los dos anteriores, aunque en este caso, lo hace a t ravés de la u tilización de un nuevo el emento, el pat rimonio. Así, Valencia Narbona y Villarroel Herrera llevan a cabo una propuesta en torno al patrimonio local para los estudiantes de $3^{\circ}$ medio so bre la historia de I a ciudad andina. Con el título "Rutas patrimoniales para la enseñanza de la historia: valoración de la Sociedad Industrial de los Andes (1904-1981)" los autores desarrollan una propuesta para la valoración del patrimonio fundamentada en la ciudad como un libro abierto, una fuete histórica q ue r efleja el pasa do y que puede s er co mprendida y a nalizada por I os estudiantes tanto en su s elementos materiales como en los elementos propios del patrimonio inmaterial. Esta propuesta, aplicada a un espacio particular en la región de Valparaíso, permite por un lado, el desarrollo de una percepción en torno al patrimonio local mucho mayor, y, por otro lado, la valoración del espacio, del entorno cercano y el fomento de la apropiación territorial por parte de los alumnos.

El úl timo ca pítulo de e sta ob ra, titulado "Para un a so ciedad i nclusiva, $\mathrm{P}$ ropuesta didáctica par a el trabajo de I a i nmigración, di scriminación e i nclusión en I as aulas chilenas" abre un nuevo apartado y desarrolla una propuesta centrada en el fomento de la reflexión en la escuela en torno al fenómeno de la inmigración en Chile. Si bien esta pr opuesta se fundamenta en el desa rrollo d el t rabajo del hi storiador y del pensamiento hi stórico mediante el anál isis, el co mentario y la r eflexión de fuentes históricas diversas, el plan de trabajo en el aula se enfoca, de manera especial, hacia el fomento de un elemento clave de la competencia histórica, la empatía histórica. De esta manera, el obj etivo general que se plantea es el de desarrollar un elemento transversal del curriculum chileno a través de la enseñanza de la historia; la inclusión de todos como ciudadadanos de una comunidad.

Esta obra presenta en su conjunto un compendio de propuestas y aproximaciones, de trabajos que nos acercan a la realidad del aula, pero que también desarrollan distintos planteamientos pedagógicos, pr oblemáticas sociales actuales y que no pi erden de vista como objetivo los distintos elementos del pensamiento histórico. Unos estudios y unos análisis que desarrollan propuestas para acercar al alumnado a unos procesos y dinámicas de aprendizaje de I a di scilpina h istórica distintos e innovadores, en ot ras palabras, unas propuestas que permiten ast os estudiantes insertarse apropiadamente en el siglo XXI y hacerlo de la mano de sus docentes en formación. 
Es por todo ello que esta obra presenta un interesante acercamiento a la enseñanza en el ám bito ch ileno, per ot ambién, unas propuestas, unos en foques y una $\mathrm{s}$ aproximaciones al as realidades del aul a que son ex trapoplables tanto en su $\mathrm{s}$ experiencias de apr endizaje, como en s us estrategias e i ncluso en I os recursos utilizados más allá de las fronteras de sus casos de estudio, a otros espacios y contextos en I os que potenciar el des arrollo y el apr endizaje de una hi storia más competencial a través del pensamiento histórico.

\begin{tabular}{l} 
Publicado bajo licencia internacional Creative Commons Attribution-NonCommercial-ShareAlike4.0 \\
(CC-BY-NC-SA 4.0). Se permite copiar, usar, distribuir, transmitir y exhibir públicamente, \\
siempre que: i) se reconozca la a utoría y la fuente original de publicación (revista, editorial y \\
URL del trabajo); ii) no se utilice con fines comerciales; iii) se mencione la existencia y \\
especificaciones de esta licencia. \\
\hline
\end{tabular} 